\title{
Mathematical Model and Solution for an Unsteady MHD Fourth Grade Fluid Flow over a Vertical Plate in a Porous Medium with Magnetic Field and Suction/Injection Effects
}

\author{
O. J. Fenuga ${ }^{1 *}$, S. J. Aroloye ${ }^{1}$, S. O. Salawu ${ }^{2}$ \\ ${ }^{1}$ Department of Mathematics, University of Lagos, Akoka, Lagos State, Nigeria \\ ${ }^{2}$ Department of Mathematics, Landmark University, Omu-Aran, Osun State, Nigeria \\ Received 21 February 2020, accepted in final revised form 18 June 2020
}

\begin{abstract}
This work investigates the mathematical model and solution for an unsteady MHD fourth grade fluid flow over a vertical plate in a porous medium with the effects of the magnetic field and suction/injection parameters using Homotopy Perturbation Method. The flow is considered to satisfy the constitutive equations of fourth grade fluid flow model and because of the Homotopy Perturbation Method used, only the momentum equation with initial and boundary conditions are solved as governing equations. After initializing stability test, the convergence of the governing equations are observed graphically using the results of Homotopy Perturbation Method with the new analytical method used by Yurusoy in literature and there is a perfect agreement in results. The impact of dimensionless second, third and fourth grade parameters with the effects of magnetic field and suction/injection parameters on the velocity field are displayed graphically and discussed. Increase in suction parameter decreases the momentum boundary layer thickness while injection parameter enhances velocity distribution in the boundary layer. Magnetic field reduces velocity throughout the boundary layer because the Lorentz force which acts as retarding force reduces the boundary layer thickness.
\end{abstract}

Keywords: Unsteady; MHD; Fourth grade fluid flow; Homotopy perturbation method; Boundary layer thickness.

(C) 2020 JSR Publications. ISSN: 2070-0237 (Print); 2070-0245 (Online). All rights reserved. doi: http://dx.doi.org/10.3329/jsr.v12i4.45551 J. Sci. Res. 12 (4), 485-498 (2020)

\section{Introduction}

Industrially and technologically, considerable attention has been given in recent years to flow problems of non-Newtonian fluids. Due to the flow behaviour of non-Newtonian fluids, their governing equations are much more complicated and highly non-linear when compared to Newtonian fluids which have few analytical solutions. Non-Newtonian fluids exhibit normal stress differences and their viscosity depends on the shear rate. These distinct features and other features of non-Newtonian fluids result in more complex equations for the fluid flow. There is no single model that clearly exhibits the properties of

\footnotetext{
*Corresponding author: ofenuga@unilag.edu.ng
} 
all non-Newtonian fluids. Arifuzzaman et al. [1] analyzed heat and mass transfer characteristics of naturally convective hydromagnetic flow of fourth grade radiative fluid resulting from vertical porous plate taking into consideration nonlinear order chemical reaction and heat generation with thermal diffusion by using finite difference scheme explicitly. Rajagopal [2] examined the stability of third grade fluid with results and discussions given on many of the interesting and challenging issues in the fluids of differential type. Arifuzzaman et al. [3] numerically investigated the MHD convective and chemically reactive unsteady micropolar fluid flow with nanoparticles through the vertical porous plate with mass diffusion, thermal radiation, radiative absorption and heat source with a flow model by using boundary layer approximation. Homotopy Analysis Method was used for the fourth grade flow of a Couette and Poiseuille flows by Siddiqui et al. [4]. The effects of some thermos-physical parameters were considered on the flow of a fourth grade or fourth order fluid in a porous space or plate by Hayat et al. [5-7]. Reza-E-Rabbi et al. [8] elaborated the heat and mass transfer analysis of casson Nano fluid flow past a stretching sheet together with MHD thermal radiation and chemical reaction effects using explicit finite difference scheme. Arifuzzaman et al. [9] modeled an unsteady natural convective and higher order chemically reactive MHD fluid flow with heat and radiation absorption effects through a vertical oscillating porous plate. Boundary layer approximation was carried out to establish a flow model of time dependent momentum, energy and diffusion balance equations using non similar technique with explicit finite difference method. In another work, anomalous features were given in the model of second order fluid by Fosdick and Rajagopal [10]. The governing differential equation is highly non-linear and the analytic solution has not been reported in literature. Also, the order of the differential equation in the case of fourth grade fluid flow was higher than that of the Navier-Stokes equations. The no-slip boundary condition is sufficient for a Newtonian fluid but for a non-Newtonian fourth grade fluid flow, it may not be sufficient and therefore needs additional conditions at the boundary. The detailed review on the boundary conditions and the existence and uniqueness of the solution for second and third grade fluids were given by Passerini and Patria [11]. Arifuzzaman et al. [12] considered the numerical investigation of MHD transient naturally convective and higher order chemically reactive Maxwell fluid with nanoparticle flow through a vertical porous plate with the effects of heat generation and radiation absorption using a boundary layer approximation to develop the flow model representing time dependent momentum, energy and concentration equations which were solved by non-similar technique explicit finite difference method. This paper has established an analytic solution based on the Homotopy Perturbation Method proposed by He [13] and found that there was no need to augment the boundary conditions for the solutions of Homotopy Perturbation Method. The Homotopy Perturbation Method was successfully applied to solve some related problems by Sobamowo and Akinshilo [14]. Arifuzzaman et al. [15] gave a theoretical work with numerical investigation of MHD transient naturally convective and higher order chemically reactive, viscoelastic fluid with nanoparticles flow through a vertical porous stretching sheet with the effect of heat generation and radiation absorption. A boundary 
layer approximation was carried out to develop the flow model representing time dependent momentum, energy and concentration equations which were solved using non similar technique of explicit finite difference method. Tan and Masuoka [16], Wang and $\mathrm{Wu}$ [17] studied some important review on fluid of differential type; new analytical solution of a fourth grade fluid past a porous plate was investigated by Yurusoy [18] and Heat transfer analysis of the steady flow of a fourth grade fluid flow was studied by Hayat et al. [19].

Homotopy Perturbation Method is a simple and total approximate analytical method. Unlike Differential Transform Method (DTM), Homotopy Analysis Method (HAM), Adomian Decomposition Method (ADM), Variation Iteration Method (VIM) etc. where the search for a particular value that will satisfy the other end of the boundary condition necessitate the use of a numerical method with the use of a software which could result in additional computational cost in the generation of solution. Homotopy Perturbation Method eliminates the "small parameter assumption" as in the traditional perturbation methods. It is a powerful method that gives acceptable analytical results with convenient convergence and stability. Therefore, in finding the approximate analytical solutions of linear and nonlinear differential equations, Homotopy Perturbation Method has gained more ground in many engineering and scientific research papers [20]. It is an approximate analytical method that can solve differential equations, difference equations and differential-difference equations, fractional differential equations, pantograph equations and inter-differential equations. It also solves nonlinear integral and differential equations without linearization, discretization, closure, restrictive assumption, perturbation, approximations and round-off error that could result in massive numerical computations. It does not require small parameters in algebraic or differential equations as in other traditional perturbation method (regular and singular perturbations). It provides excellent approximations to the solutions of non-linear equations with higher accuracy. Marinca et al. [21] used an optimal homotopy assumption method to solve a steady flow of a fourth grade fluid past a porous plate. An optimal solution for the flow of the fourth grade fluid with partial slip was investigated by Islam et al. [22] and the effects of partial slip on fourth grade fluid with variable viscosity was investigated by Nadeem et al. [23].

This work provides the mathematical model and solution for an MHD fourth grade fluid flow past a vertical porous plate with magnetic and suction/injection effects. The motivation comes from the desire to understand the shear thinning and thickening property of non-Newtonian fluids in the presence of magnetic field and suction /injection. This paper extends the works of Marinca et al. [21], Islam et al. [22] and Nadeem et al. [23] to include the effects of magnetic field and suction/ injection parameters with semianalytical solution via Homotopy Perturbation Method to solve the higher order nonlinear differential equations describing the fluid flow.

\section{Nomenclature}

MHD - Magnetohydrodynamics

$\frac{\mathrm{d}}{\mathrm{dt}}$ is the material time derivative defined by $\frac{d}{d t}=\frac{\partial}{\partial t}+(\mathrm{V} . \nabla)$ 
$X$ denotes the vector product

$\mathbf{T}$ is the Cauchy stress tensor for an incompressible fourth grade fluid,

$\mathbf{J}$ is the current density, B is the magnetic induction

$\boldsymbol{R}=\left(R_{x}, R_{y}, R_{z}\right)$ is the Darcy's resistance due to the porous media in the $x, y$, and $z$ axes respectively

$u(y, t)$ represents the fluid velocity field in the $x$ direction

$V_{0}>0$ is the suction velocity and $V_{0}<0$ is the injection velocity

$\mathbf{B}_{\mathbf{0}}$ is the applied magnetic field and $\mathbf{b}$ is the induced magnetic field

$\sigma$ is the electrical conductivity of the fluid

$\mathrm{P}$ is pressure, $\mathrm{I}$ is the identity matrix and $\mu$ is the viscosity

$\mathbf{A}_{1}, \mathbf{A}_{2}, \mathbf{A}_{\mathbf{3}}$ and $\mathbf{A}_{\mathbf{4}}$ are Rilvlin-Ericksen tensors

$\rho$ denotes fluid density

$U_{0}$ is the mainstream velocity

$\alpha_{1}, \alpha_{2}, \beta_{1}, \beta_{2}, \beta_{3}, \gamma_{1}, \gamma_{2,}, \gamma_{3}, \gamma_{4}, \gamma_{5}, \gamma_{6}, \gamma_{7}$, and $\gamma_{8}$ are the material parameters

$\alpha$ is the second grade parameter, $\beta$ is the third grade parameter and $\gamma$ is the fourth grade parameter.

\section{The Constitutive Equations for Fourth Grade Flow}

Considers an unsteady, MHD, incompressible, electrically conducting fourth grade fluid flow (a subclass of differential type fluids) which is an important class of non-Newtonian fluids that occupies region $y>0$ in a porous medium. A Cartesian frame of reference along $x$-axis is chosen as the direction of fluid flow, parallel to the porous plate and the $y$ axis is perpendicular to it. At $\mathrm{t}=0$, the fluid is bounded by an infinite porous plate. A transverse uniform magnetic field $\mathbf{B}=\left(0, B_{0}, 0\right)$ is applied at the surface of the plate. Due to porous character of the plates, there is a cross-flow of the fluid with a constant velocity $V_{0}$. The velocity field of the fluid flow is a functions of $y$ and $t$ only. The plate is coinciding with the plane at $y=0$. Unsteady motion of the conducting fluid through a porous medium is governed by the conservation laws of momentum and mass as stated below:

$$
\begin{array}{lc}
\text { Continuity equation } & \nabla \cdot \mathbf{V}=0 \\
\text { Momentum equation } & \frac{\mathrm{d} \mathbf{V}}{\mathrm{dt}}=\nabla \cdot \mathbf{T}+\mathbf{J} \times \mathbf{B}+
\end{array}
$$

Where $\frac{\mathrm{d}}{\mathrm{dt}}$ is the material time derivative defined by $\frac{d}{d t}=\frac{\partial}{\partial t}+(\mathbf{V} . \nabla)$

$X$ denotes the vector product, $\mathbf{T}$ is the Cauchy stress tensor for an incompressible fourth grade fluid, $\mathbf{J}$ is the current density, B is the magnetic induction and $\boldsymbol{R}=\left(R_{x}, R_{y}, R_{z}\right)$ is the Darcy's resistance due to the porous media in the $x, y, z$ respectively. For the flow model under investigation, we consider a velocity field $\mathbf{V}$ of the form

$$
\mathbf{V}=\left(u(y, t),-V_{0}, 0\right)
$$


Where $u(y, t)$ represents the fluid velocity field in the $x$ direction, $V_{0}>0$ is the suction velocity and $V_{0}<0$ is the injection velocity. This velocity field identically satisfies the continuity equation (1). Thus, the disturbance in the fluid is a function of $y$ and $t$ only.

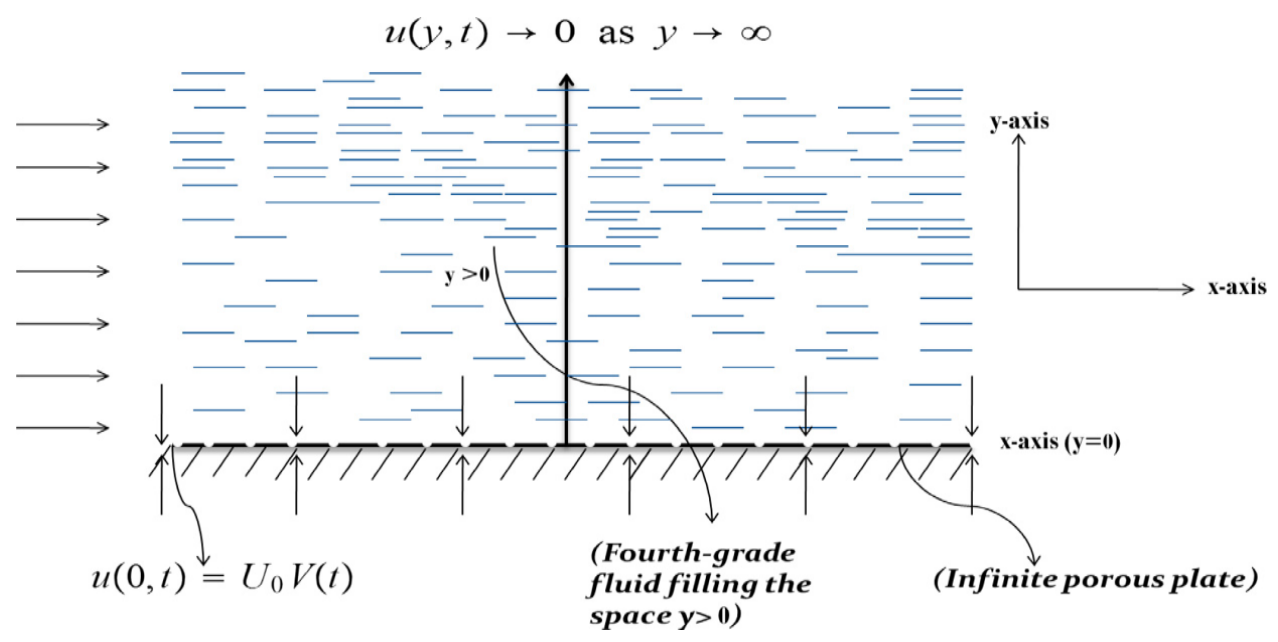

Fig. 1. Geometry of the physical model and coordinate system.

The current density given by $\mathbf{J}$ and $\mathbf{B}$ gives the total magnetic field such that $\mathbf{B}=\mathbf{B}_{\mathbf{0}}+\mathbf{b}$ where $\mathbf{B}_{\mathbf{0}}$ is the applied magnetic field and $\mathbf{b}$ is the induced magnetic field.

In the analysis, the fluid is electrically conducting and a magnetic field is applied in the transverse direction to the flow. With the low magnetic Reynolds number, the induced electric and magnetic fields can be neglected and thus the magnetic force $\mathbf{J} \times \mathbf{B}$ becomes

$$
\mathbf{J} \times \mathbf{B}=\sigma(\mathbf{V} \times \mathbf{B}) \times \mathbf{B}=-\sigma B_{0}^{2} \mathbf{V}
$$

$\sigma$ is the electrical conductivity of the fluid

The Cauchy stress tensor for a fourth grade fluid which satisfies the constitutive equation is

$$
\begin{aligned}
& \mathbf{T}=-\mathrm{PI}+\mu \mathbf{A}_{\mathbf{1}}+\alpha_{1} \mathbf{A}_{\mathbf{1}}+\alpha_{2} \mathbf{A}_{\mathbf{1}}^{2}+\beta_{2}\left(\mathbf{A}_{\mathbf{2}} \mathbf{A}_{\mathbf{1}}+\mathbf{A}_{\mathbf{1}} \mathbf{A}_{\mathbf{2}}\right)+\beta_{3}\left(\operatorname{tr} \mathbf{A}_{\mathbf{1}}^{2}\right) \mathbf{A}_{\mathbf{1}}+\gamma_{1} \mathbf{A}_{\mathbf{4}}+ \\
& \gamma_{2}\left(\mathbf{A}_{\mathbf{3}} \mathbf{A}_{\mathbf{1}}+\mathbf{A}_{\mathbf{1}} \mathbf{A}_{\mathbf{3}}\right)+\beta_{1} \mathbf{A}_{\mathbf{3}}+\gamma_{3} \mathbf{A}_{\mathbf{2}}^{2}+\gamma_{4}\left(\mathbf{A}_{\mathbf{1}} \mathbf{A}_{\mathbf{1}}^{2}+\mathbf{A}_{\mathbf{1}}^{2} \mathbf{A}_{\mathbf{1}}\right)+\gamma_{5}\left(\operatorname{tr} \mathbf{A}_{\mathbf{2}}\right) \mathbf{A}_{\mathbf{2}}+\gamma_{6}\left(\operatorname{tr} \mathbf{A}_{2}\right) \mathbf{A}_{\mathbf{1}}^{2}+ \\
& {\left[\gamma_{7}\left(\operatorname{tr} \mathbf{A}_{\mathbf{3}}\right)+\gamma_{\mathbf{8}}\left(\operatorname{tr} \mathbf{A}_{\mathbf{2}} \mathbf{A}_{\mathbf{1}}\right)\right] \mathbf{A}_{\mathbf{1}}}
\end{aligned}
$$

Where $P$ is pressure, $I$ is the identity matrix, $\mu$ is the viscosity,

$\alpha_{1}, \alpha_{2}, \beta_{1}, \beta_{2}, \beta_{3}, \gamma_{1}, \gamma_{2}, \gamma_{3}, \gamma_{4}, \gamma_{5}, \gamma_{6}, \gamma_{7}$, and $\gamma_{8}$ are the material parameters;

$\mathbf{A}_{1}, \mathbf{A}_{2}, \mathbf{A}_{\mathbf{3}}$ and $\mathbf{A}_{\mathbf{4}}$ are Rilvlin-Ericksen tensors. The tensors are defined as

$$
\begin{aligned}
& \mathbf{A}_{\mathbf{1}}=(\nabla \mathbf{V})+(\nabla \mathbf{V})^{T} \\
& \mathbf{A}_{\mathrm{n}}=\frac{\mathbf{d} \mathbf{A}_{\mathrm{n}-\mathbf{1}}}{d t}+\mathbf{A}_{\mathrm{n}-\mathbf{1}}(\nabla \mathbf{V})+(\nabla \mathbf{V})^{T} \mathbf{A}_{\mathrm{n}-\mathbf{1}}, \quad \mathrm{n}>1
\end{aligned}
$$

Where $\nabla$ is the gradient operator, $T$ denotes the transpose of the resultant matrix. For the model (5) above, when $\alpha_{i}=0, \beta_{i}=0$ and $\gamma_{i}=0$, the fluid is Newtonian. If $\alpha_{i} \neq 0$, $\beta_{\mathrm{i}}=0$ and $\gamma_{\mathrm{i}}=0$; it is equivalent to second grade fluid; if $\alpha_{\mathrm{i}} \neq 0, \beta_{\mathrm{i}} \neq 0$ and $\gamma_{\mathrm{i}}=0$, it 
is equivalent to the third grade fluid and if $\alpha_{i} \neq 0, \beta_{i} \neq 0$, and $\gamma_{i} \neq 0$; then it is equivalent to a fourth grade fluid.

For the fourth grade fluid flow past a porous vertical plate with suction or injection, the $x$ and $y$-axes are respectively chosen to be parallel and normal to the plate while the velocity field depends on $y$ only. The complete sets of governing equations for the fourth grade fluid flow in the presence of magnetic field are:

$$
\begin{aligned}
& \operatorname{div} \mathbf{V}=0 \\
& \rho \frac{d \mathbf{V}}{d t}=\operatorname{div} \mathbf{T}+\mathbf{J} \times \mathbf{B}
\end{aligned}
$$

where $\rho$ denotes fluid density

We seek a velocity field $\mathbf{V}$ of the form

$$
\mathbf{V}=\left(u(y),-V_{0}, 0\right)
$$

Where $u(y)$ represent the fluid velocity field in the $x$ direction, $V_{0}>0$ is the suction velocity and $V_{0}<0$ is the injection velocity. The velocity field satisfies the continuity eq. (8). Thus, the disturbance in the fluid is a function of $y$

Using eqs. (6), (7), (8) and (10), eq. (5) gives stress components as follows

$$
\begin{aligned}
& \tau_{x y}=-p+\alpha_{2}\left(\frac{d u}{d y}\right)^{2}-2 \beta_{2} V_{0} \frac{d u}{d y} \frac{d^{2} u}{d y^{2}}+2 \gamma_{2} V_{0}^{2} \frac{d u}{d y} \frac{d^{3} u}{d y^{3}}+\gamma_{3} V_{0}^{2}\left(\frac{d^{2} u}{d y^{2}}\right)^{2}+2 \gamma_{6}\left(\frac{d u}{d y}\right)^{6} \\
& \tau_{x y}=\mu \frac{d u}{d y}+\alpha_{1} V_{0} \frac{d^{2} u}{d y^{2}}-\beta_{1} V_{0}^{2} \frac{d^{3} u}{d y^{3}}+2\left(\beta_{1}+\beta_{2}\right)\left(\frac{d u}{d y}\right)^{3}-\gamma_{1} V_{0}^{3} \frac{d^{4} u}{d y^{4}}+ \\
& \left(6 \gamma_{2}+2 \gamma_{3}+2 \gamma_{4}+2 \gamma_{5}+6 \gamma_{7}+2 \gamma_{8}\right) V_{0}\left(\frac{d u}{d y}\right)^{2} \frac{d^{2} u}{d y^{2}} \\
& \tau_{y y}=-p+\left(2 \alpha_{1}+\alpha_{2}\right)\left(\frac{d u}{d y}\right)^{2}-2\left(\beta_{1}+\beta_{2}\right) V_{0} \frac{d u}{d y} \frac{d^{2} u}{d y^{2}}+6 \gamma_{1} V_{0}^{2} \frac{d}{d y}\left(\frac{d u}{d y} \frac{d^{2} u}{d y^{2}}\right)+ \\
& 2\left(\gamma_{1}+\gamma_{2}\right) V_{0}^{2} \frac{d^{3} u}{d y^{3}} \frac{d u}{d y}+\gamma_{3} V_{0}^{2}\left(\frac{d^{2} u}{d y^{2}}\right)^{2}+2\left(2 \gamma_{3}+2 \gamma_{4}+2 \gamma_{5}+6 \gamma_{6}\right)\left(\frac{d u}{d y}\right)^{4} \\
& \tau_{z z}=-p \\
& \tau_{x z}=\tau_{z y}=0 \\
& \tau_{x y}=\tau_{y x}, \tau_{x z}=\tau_{z x}, \tau_{y z}=\tau_{z y}
\end{aligned}
$$

Substituting equations (11-16) in equation (9) gives

$$
\begin{aligned}
& -\rho V_{0} \frac{d u}{d y}=\mu \frac{d^{2} u}{d y^{2}}+\alpha_{1} V_{0} \frac{d^{3} u}{d y^{3}}-\beta_{1} V_{0}^{2} \frac{d^{4} u}{d y^{4}}+6\left(\beta_{1}+\beta_{2}\right)\left(\frac{d u}{d y}\right)^{2} \frac{d^{2} u}{d y^{2}}-\gamma_{1} V_{0}^{3} \frac{d^{5} u}{d y^{5}}- \\
& \left(6 \gamma_{2}+2 \gamma_{3}+2 \gamma_{4}+2 \gamma_{5}+6 \gamma_{7}+2 \gamma_{8}\right) V_{0} \frac{d}{d y}\left(\frac{d u}{d y}\right)^{2} \frac{d^{2} u}{d y^{2}}
\end{aligned}
$$

The boundary conditions on $u$ are

$$
\begin{aligned}
& u(0)=0 \\
& u(y) \rightarrow U_{0}, \quad \frac{d^{n} u}{d y^{n}} \rightarrow 0 \text { as } y \rightarrow \infty \text { for } n=1,2,3
\end{aligned}
$$


where $U_{0}$ is the mainstream velocity, $\alpha$ is the second grade parameter, $\beta$ is the third grade parameter and $\gamma$ is the fourth grade parameter.

Introducing the following non-dimensional quantities

$$
u=\frac{u}{U_{0}}, y=\frac{U_{0} y}{v}, V_{0}=\frac{V_{0}}{U_{0}},
$$

Using (20) in (17) - (19), then the governing equation is obtained as

$$
\begin{aligned}
& \frac{d^{2} u}{d y^{2}}+V_{0} \frac{d u}{d y}-\alpha_{1} V_{0} \frac{d^{3} u}{d y^{3}}+\beta_{1} V_{0}^{2} \frac{d^{4} u}{d y^{4}}+\beta\left(\frac{d u}{d y}\right)^{2} \frac{d^{2} u}{d y^{2}}-\gamma_{1} V_{0}^{3} \frac{d^{5} u}{d y^{5}}- \\
& \Gamma V_{0}\left(2 \frac{d u}{d y}\left(\frac{d^{2} u}{d y^{2}}\right)^{2}+\left(\frac{d u}{d y}\right)^{2} \frac{d^{2} u}{d y^{2}}\right)-M^{2} u=0
\end{aligned}
$$

with the dimensionless boundary conditions

$$
u(0) \rightarrow 0, \text { aty }=0, u(1) \rightarrow 1, \frac{d^{n} u}{d y^{n}} \rightarrow 0 \text { as } y \rightarrow \infty \text { for } n=1,2,3
$$

The dimensionless parameters in eq. (21) are

$$
\begin{aligned}
& \alpha=\frac{\alpha_{1} U_{0}^{2}}{\rho v^{2}}, \beta_{1}=\frac{\beta_{1} U_{0}^{4}}{\rho v^{3}}, \gamma_{1}=\frac{\gamma_{1} U_{0}^{6}}{\rho v^{4}}, \beta=\frac{6\left(\beta_{1}+\beta_{1}\right) U_{0}^{4}}{\rho v^{2}}, \Gamma=6\left(3 \gamma_{2}+\gamma_{3}+\gamma_{4}+\gamma_{5}+\gamma_{7}+\right. \\
& \left.\gamma_{8}\right) \frac{U_{0}^{6}}{\rho v^{4}}
\end{aligned}
$$

The fifth order differential eq. (21) subject to the boundary conditions (22) is solved using Homotopy Perturbation Method (HPM).

\subsection{Homotopy perturbation method and convergence}

Consider a system of nonlinear differential equations given as

$$
A(U)-f(r)=0, r \in \Omega
$$

with the boundary conditions

$$
B\left(u, \frac{\partial u}{\partial \eta}\right)=0, r \in \Gamma
$$

where $\mathrm{A}$ is a general differential operator, B is a boundary operator, $f(r)$ is a known analytical function and $\Gamma$ is the boundary of the domain $\Omega$.

The operator A can be divided into two parts, $\mathrm{L}$ and $\mathrm{N}$, where $\mathrm{L}$ is a linear operator, $\mathrm{N}$ is a non-linear operator. Then, eq. (24) can therefore be written as

$$
L(u)+N(u)-f(r)=0
$$

By the Homotopy technique, an homotopy $U(r, p): \Omega \times[0,1] \rightarrow R$ can be constructed which satisfies

Or

$$
H(U, p)=(1-p)\left[L(U)-L\left(U_{0}\right)\right]+p[A(U)-f(r)]=0, p \epsilon[0,1]
$$

$$
H(U, p)=L(U)-L\left(U_{0}\right)+p L(U)+p[N(U)-f(r)]=0 \quad p \epsilon[0,1]
$$


In eqs. (27) or (28), $p \in[0,1]$ is an embedding parameters, $u$ is an initial approximation of (24) which satisfies the boundary condition.

Also, from eqs. (27) or (28),

$$
H(U, p)=L(U)-L\left(U_{0}\right)=0 \operatorname{or} H(U, p)=A(U)-f(r)=0
$$

The changing process of $\mathrm{p}$ from zero to unity is like $U(r, p)$ changing from $u_{0}(0)$ to $u(r)$. This is referred to as Homotopy in topology. Using the embedding parameter $\mathrm{p}$ as a small parameter, the solution of eq. (27) or (28) is assumed to be written in power series as

$$
U=U_{0}+p U_{1}+p^{2} U_{2}+\cdots
$$

It should be noted that all the values of $\mathrm{p}$ are between 0 and 1 but $\mathrm{p}=1$ produces the best result. Therefore, setting $\mathrm{p}=1$, results in the approximate solution of (24) gives

$$
U=\lim _{p \rightarrow 1} U=U_{0}+U_{1}+U_{2}+\cdots
$$

The convergent series (31) is a combination of Homotopy and Perturbation Methods. Hence, the method is called Homotopy Perturbation Method (HPM).

According to Homotopy Perturbation Method (HPM), one can construct a homotopy for eq. (21) as

$$
\begin{aligned}
& H(p, y)=(1-p) \gamma_{1}\left(V_{0}^{3} \frac{d^{5} u}{d y^{5}}\right)+p\left[\frac{d^{2} u}{d y^{2}}+V_{0} \frac{d u}{d y}-\alpha_{1} V_{0} \frac{d^{3} u}{d y^{3}}+\beta_{1} V_{0}^{2} \frac{d^{4} u}{d y^{4}}+\right. \\
& \left.\beta\left(\frac{d u}{d y}\right)^{2} \frac{d^{2} u}{d y^{2}}-\gamma_{1} V_{0}^{3} \frac{d^{5} u}{d y^{5}}-\gamma V_{0} 2 \frac{d u}{d y}\left(\frac{d^{2} u}{d y^{2}}\right)^{2}+\left(\frac{d u}{d y}\right)^{2} \frac{d^{2} u}{d y^{2}}\right]=0
\end{aligned}
$$

The power series of velocity field is given as

$$
u=u_{0}+u_{1}+u_{2}+\cdots .
$$

Then, substituting eq. (33) into eq. (32) using perturbation method yields

$$
\begin{array}{ll}
p^{0}: & \frac{d^{5} u_{0}}{d y^{5}}= \\
p^{1}: & \frac{d^{5} u_{1}}{d y^{5}}+\frac{d^{2} u_{0}}{d y^{2}}+V_{0} \frac{d u_{0}}{d y}-\alpha_{1} V_{0} \frac{d^{3} u_{0}}{d y^{3}}+\beta_{1} V_{0}^{2} \frac{d^{4} u_{0}}{d y^{4}}-M^{2} u_{0}+ \\
& \beta\left(\frac{d u_{0}}{d y}\right)^{2} \frac{d^{2} u_{0}}{d y^{2}}-\gamma V_{0}\left(2 \frac{d u_{0}}{d y}\left(\frac{d^{2} u_{0}}{d y^{2}}\right)^{2}+\left(\frac{d u_{0}}{d y}\right)^{2} \frac{d^{2} u_{0}}{d y^{2}}\right)=0 \\
p^{2}: \quad & \frac{d^{5} u_{2}}{d y^{5}}+\frac{d^{2} u_{1}}{d y^{2}}+V_{0} \frac{d u_{1}}{d y}-\alpha_{1} V_{0} \frac{d^{3} u_{1}}{d y^{3}}+\beta_{1} V_{0}^{2} \frac{d^{4} u_{1}}{d y^{4}}+\beta\left(\frac{d u_{0}}{d y}\right)^{2} \frac{d^{2} u_{0}}{d y^{2}}+ \\
& 2 \beta \frac{d u_{0}}{d y} \frac{d u_{1}}{d y} \frac{d^{2} u_{0}}{d y^{2}}-\gamma V_{0}\left(2 \frac{d u_{0}}{d y}\left(\frac{d^{2} u_{0}}{d y^{2}}\right)+\frac{d u_{0}}{d y} \frac{d u_{0}}{d y}\left(\frac{d^{2} u_{1}}{d y^{2}}\right)^{2}+\left(\frac{d u_{0}}{d y}\right)^{2} \frac{d^{2} u_{0}}{d y^{2}}+\right. \\
& \left.\frac{d u_{0}}{d y} \frac{d u_{1}}{d y} \frac{d^{2} u_{0}}{d y^{2}}\right)-M^{2} u_{1}=0
\end{array}
$$




$$
\begin{aligned}
p^{3}: & \frac{d^{5} u_{3}}{d y^{5}}+\frac{d^{2} u_{2}}{d y^{2}}+V_{0} \frac{d u_{2}}{d y}-\alpha_{1} V_{0} \frac{d^{3} u_{2}}{d y^{3}}+\beta_{1} V_{0}^{2} \frac{d^{4} u_{2}}{d y^{4}}+\beta\left(\frac{d u_{1}}{d y}\right)^{2} \frac{d^{2} u_{0}}{d y^{2}}+ \\
& \beta \frac{d u_{1}}{d y}\left(\frac{d u_{0}}{d y}\right)^{2} \frac{d^{2} u_{1}}{d y^{2}}+2 \beta \frac{d u_{1}}{d y} \frac{d u_{1}}{d y} \frac{d^{2} u_{0}}{d y^{2}}-\gamma V_{0}\left(2 \frac{d u_{0}}{d y}\left(\frac{d^{2} u_{0}}{d y^{2}}\right)+\frac{d u_{0}}{d y}\left(\frac{d^{2} u_{1}}{d y^{2}}\right)^{2}+\right. \\
& \left.\left(\frac{d u_{1}}{d y}\right)^{2} \frac{d^{2} u_{0}}{d y^{2}}+\frac{d u_{0}}{d y} \frac{d u_{1}}{d y} \frac{d^{2} u_{1}}{d y^{2}}+\frac{d u_{1}}{d y} \frac{d u_{1}}{d y} \frac{d^{2} u_{0}}{d y^{2}}\right)-M^{2} u_{2}=0
\end{aligned}
$$

The boundary conditions for Eqs. (34)-(37) are

$$
\begin{aligned}
& u_{0}(0) \rightarrow 0, \text { at } y=0, u_{0}(1) \rightarrow 1, \frac{d^{n} u_{0}}{d y^{n}} \rightarrow 0 \text { as } y \rightarrow \infty \text { for } n=1,2,3 \\
& u_{1}(0) \rightarrow 0 \text {, at } y=0, u_{0}(1) \rightarrow 0, \frac{d^{n} u_{0}}{d y^{n}} \rightarrow 0 \text { as } y \rightarrow \infty \text { for } n=1,2,3 \\
& u_{2}(0) \rightarrow 0 \text {, at } y=0, u_{2}(1) \rightarrow 0, \frac{d^{n} u_{2}}{d y^{n}} \rightarrow 0 \text { as } y \rightarrow \infty \text { for } n=1,2,3 \\
& u_{3}(0) \rightarrow 0 \text {, at } y=0, u_{3}(N) \rightarrow 0, \frac{d^{n} u_{0}}{d y^{n}} \rightarrow 0 \text { as } y \rightarrow \infty(N) \text { for } n=1,2,3
\end{aligned}
$$

Solving eq. (34) and applying the boundary condition (38) results gives

$$
u_{0}(y)=\frac{y^{4}}{N^{4}}-\frac{4 y^{3}}{N^{3}}+\frac{6 y^{2}}{N^{3}}-\frac{4 y}{N^{3}}
$$

Solving eq. (35) and applying the boundary condition (38) gives

$$
\begin{aligned}
& u_{1}(y)= \\
& \frac{(3 \beta+1)^{2} y^{9}}{23 N^{4}}-\frac{4\left(V_{0}+1+\alpha\right)^{5} y^{10} y^{3}}{N^{3}}+\frac{4(M n+1+\alpha)^{5} y^{3}}{N^{3}}+\frac{\left(7 \alpha_{1}+1\right)^{2}}{23 N^{4}}+ \\
& \frac{6 \frac{4(3 \beta+M n+\alpha)^{5} y^{3}}{N^{3}} y^{2}+\frac{4(M n \beta+1+\alpha)^{5} y^{10} y^{3}}{N^{3}}}{N^{3}}-\frac{4 \frac{4(3 \beta+1+\alpha)^{5} y^{10} y^{3}}{N^{3}} y}{N^{3}}+\frac{4(3 \beta+1+\alpha)^{5} y^{10} y^{3}+\frac{4(3 \alpha+1+\alpha)^{5} y^{4}}{N^{3}}}{N^{3}}
\end{aligned}
$$

Also, solving eq. (36) and applying the boundary condition (40) gives

$$
\begin{aligned}
& u_{2}(y)= \\
& \frac{(G r+1)^{2} y^{9}}{23 N^{4}}-\frac{(5 \beta+1)^{2} y^{9}}{23 N^{4}}-\frac{4\left(V_{0}+6+\alpha\right)^{5} y^{10} y^{3}}{N^{3}}+\frac{4(M n+V+\alpha)^{5} y^{3}}{N^{3}}+\frac{\left(7 \alpha_{1}+1\right)^{2}}{23 N^{4}}+ \\
& \frac{6 \frac{4(3 \beta+M n+\alpha)^{5} y^{3}}{N^{3}} y^{2}+\frac{4(M n \beta+1+\alpha)^{2} y^{10} y^{3}}{N^{2}}}{N^{2}}-\frac{4 \frac{4(3 \beta+1+\alpha)^{5} y^{10} y^{3}}{N^{5}} y}{N^{9}}+ \\
& \frac{4(3 \beta+1+\alpha)^{5} y^{10} y^{3}+\frac{4(3 \alpha+G r+\alpha)^{5} y^{4}}{N^{3}}}{N^{3}} \frac{4\left(V_{0}+1+\alpha\right)^{5} y^{4}}{N^{3}}+\frac{4(M n+1+\alpha)^{5} y^{3}}{N^{3}} \frac{4\left(V_{0}+1+\alpha\right)^{6} y^{10^{3}}}{N^{4}}+\frac{4(M n+1+\alpha)^{5} y^{3}}{N^{51}}+ \\
& \frac{(3 \beta+1)^{2} y^{9}}{23 N^{4}}-\frac{4\left(V_{0}+1+\alpha\right)^{5} y^{10} y^{3}}{N^{3}}+\frac{4(M n+1+\alpha)^{5} y^{3}}{N^{3}}+(M n+1+\alpha)^{5} y^{3} \frac{\left(7 \alpha_{1}+1\right)^{2}}{23 N^{4}}+ \\
& \frac{6 \frac{4(3 \beta+M n+\alpha)^{5} y^{3}}{N^{3}} y^{2}+\frac{4(M n \beta+1+\alpha)^{5} y^{10} y^{3}}{N^{3}}}{N^{3}}-\frac{4 \frac{4(3 \beta+1+\alpha)^{5} y^{10} y^{3}}{N^{3}} y}{N^{3}}+\frac{4(3 \beta+1+\alpha)^{5} y^{10} y^{3}+\frac{4(3 \alpha+1+\alpha)^{5} y^{4}}{N^{3}}}{N^{3}}
\end{aligned}
$$

In the same way, $u_{3}(y)$ in eq. (37) is obtained using the boundary conditions (41) as 


$$
\begin{aligned}
& u_{3}(y)=\frac{(G r+1)^{2} y^{9}}{23 N^{4}} u-\frac{(5 \beta+1)^{2} y^{9}}{23 N^{4}}-\frac{4\left(V_{0}+6+\alpha\right)^{5} y^{10} y^{3}}{N^{3}}+\frac{4(M n+G r+\alpha)^{5} y^{3}}{N^{3}}+\frac{\left(7 \alpha_{1}+1\right)^{2}}{23 N^{4}}+ \\
& \frac{6 \frac{4(3 \beta+M n+\alpha)^{5} y^{3}}{N^{3}} y^{2}+\frac{4(M n \beta+1+\alpha)^{2} y^{10} y^{3}}{N^{2}}}{N^{2}}-\frac{4 \frac{4(3 \beta+1+\alpha)^{5} y^{10} y^{3}}{N^{5}} y}{N^{9}}+\frac{4(3 \beta+1+\alpha)^{5} y^{10} y^{3}+\frac{4(3 \alpha+G r+\alpha)^{5} y^{4}}{N^{3}}}{N^{3}} \frac{4\left(V_{0}+1+\alpha\right)^{5} y^{4}}{N^{3}}+ \\
& \frac{4(M n+1+\alpha)^{5} y^{3}}{N^{3}} \frac{4\left(V_{0}+1+\alpha\right)^{6} y^{10^{3}}}{N^{4}}+\frac{4(M n+1+\alpha)^{5} y^{3}}{N^{51}}+\frac{\left(7 \gamma_{1}+1\right)^{2}}{23 N^{4}} \frac{\left(7 \alpha_{1}+1\right)^{2}}{23 N^{4}}+ \\
& \frac{6 \frac{4(3+M n+\alpha)^{5} y^{3}}{N^{3}} y^{2}+\frac{4\left(M n \beta+V_{0}+\alpha\right)^{5} y^{4}}{N^{3}}}{N^{3}}-\frac{4 \frac{4\left(3 M n+V_{0}+\alpha\right)^{5} y^{3}}{N^{6}} y}{N^{3}}+\frac{4(4 \beta+1+\alpha)^{5} y^{5}+\frac{4(3 \alpha+G r+\alpha)^{5} y^{4}}{N^{3}}+}{N^{10}}+ \\
& \frac{4(4 \beta+1+\alpha)^{5} y^{3}+\frac{4(6 G r \alpha+G r+\alpha)^{5} y^{4}}{N^{10}}}{N^{3}}+\frac{\left(7 \gamma_{1}+1\right)^{2}}{23 N^{4}} \frac{\left(7 \alpha_{1}+1\right)^{2}}{23 N^{4}}+\frac{6 \frac{4(3+G r M n+\alpha)^{6} y^{3}}{N^{3}} y^{2}+\frac{17\left(M n \beta+V_{0}+\alpha\right)^{5} y}{N^{3}}}{N^{3}}- \\
& \frac{4 \frac{4\left(3 M n+V_{0}+\alpha\right)^{5} y^{3}}{N^{6}} y}{N^{3}}
\end{aligned}
$$

Other subsequent solutions are too long to be displayed in this paper and so are in the simulated results shown graphically in the next section.

\section{Numerical Results and Discussion}

There is an excellent agreement when the results of the Homotopy Perturbation Method (HPM) of the present work in the absence of unsteady parameter, slip and convective boundary conditions is plotted graphically in Fig. 2 using the default values $\gamma=1, \beta=$ $2, \gamma_{1}=0.5, \beta_{1}=0.8, M n=0.6, \alpha=2.5, V_{0}=0.8$ and compared respectively with the numerical method and new analytical solution of results of Hayat [19] and Yususoy [18].

Fig. 3 shows the effects of suction parameter $V_{0}$ on the velocity profile. Suction decreases the flow velocity at the surface of the plate while injection increases the flow velocity at the surface of the plate as shown in Fig. 4. It was shown that velocity profile decreases with increase in suction parameter because momentum boundary layer thickness decreases with a rise in suction velocity. The damping effect of suction on the fluid flow is attributed to the fact that the heated fluid is being pushed towards the plate with the action of the buoyancy force to resist the fluid flow as a result of high influence of viscosity. On the other hand, injection parameter enhances velocity distribution with the boundary layer. These results reveal that suction on the plate can be used to control the boundary layer thickness.

Fig. 5 shows the influence of magnetic field parameter $M$ on the velocity profiles. It was observed that the velocity profiles decreases with increase in the values of the magnetic field parameter $M$. The imposition of magnetic field in an electrically conducting fluid induces a drag-like force known as Lorentz force on the flow field which acts against the fluid flow and slows down its motion. Thus, Lorentz force increases as magnetic field parameter $M$ increases and so dampens the velocity profiles. The presence of magnetic field reduces the velocity throughout the boundary layer which is in conformity with the fact that the Lorentz force (magnetic force) acts as a retarding force which significantly reduced the momentum boundary layer thickness. 
In order to describe the influence of the third grade parameter $\beta_{1}$ and fourth grade parameter $\gamma$ on the flow model, the velocity profiles have been plotted in Figs. 6 and 7 . The results revealed that both third grade $\beta_{1}$ and fourth grade $\gamma$ parameters have opposite roles on the structure of the velocity. Increase in third grade parameter $\beta_{1}$ decreased the velocity field which indicates the shear thickening behavior of the fluid. However, increase in fourth grade parameter $\gamma$ increased the velocity profile which indicates the shear thinning property of the fluid. In Fig. 8, the fluid velocity decreases as second grade parameter increases. Table 1 shows the effect of the various embedded flow parameters on $u(y)$ and $u^{\prime}(y)$. Table 1 shows that as the distance from plate increases, the fluid velocity increases which satisfy the boundary condition and shows an excellent agreement with the Homotopy Perturbation Method (HPM) of the present work and numerical methods by Yurusoy [18].

Table 1. The results of Homotopy Perturbation Method (HPM) of the present work and Numerical Method for $u(y)$ and $u^{\prime}(y)$ of Yurusoy [18] in literature.

\begin{tabular}{|c|c|c|c|c|c|c|}
\hline & \multicolumn{3}{|c}{$u(y)$} & \multicolumn{3}{|c|}{$u^{\prime}(y)$} \\
\hline$y$ & HPM & Numerical & DIFF & HPM & Numerical & DIFF \\
\hline 0.00 & 0.000000000 & 0.000000000 & 0.000000000 & 0.202330464 & 0.202330464 & 0.000000000 \\
\hline 0.50 & 0.095008864 & 0.095031320 & 0.000022456 & 0.178419900 & 0.178435574 & 0.000015674 \\
\hline 1.00 & 0.178986457 & 0.179043189 & 0.000056732 & 0.158173350 & 0.158185695 & 0.000012345 \\
\hline 1.50 & 0.253689000 & 0.253756740 & 0.000067740 & 0.141129079 & 0.141174440 & 0.000045361 \\
\hline 2.00 & 0.320635949 & 0.320690678 & 0.000054729 & 0.126923781 & 0.127002344 & 0.000078563 \\
\hline 2.50 & 0.381103945 & 0.381169679 & 0.000065734 & 0.115262239 & 0.115295004 & 0.000032765 \\
\hline 3.00 & 0.436277311 & 0.436339652 & 0.000062341 & 0.105645333 & 0.105712788 & 0.000067455 \\
\hline 3.50 & 0.487173579 & 0.487186257 & 0.000012678 & 0.097920387 & 0.097954603 & 0.000034216 \\
\hline 4.00 & 0.534488560 & 0.534554294 & 0.000065734 & 0.091734408 & 0.091757864 & 0.000023456 \\
\hline 4.50 & 0.579149879 & 0.579166465 & 0.000016586 & 0.086870717 & 0.086896287 & 0.000025570 \\
\hline 5.00 & 0.621627274 & 0.621640729 & 0.000013455 & 0.083110820 & 0.083176573 & 0.000065753 \\
\hline 5.50 & 0.662470404 & 0.662505870 & 0.000035466 & 0.080380526 & 0.080434699 & 0.000054173 \\
\hline 6.00 & 0.702149667 & 0.702215201 & 0.000065534 & 0.078477871 & 0.078532197 & 0.000054326 \\
\hline 6.50 & 0.741112825 & 0.741158457 & 0.000045632 & 0.077286774 & 0.077352664 & 0.000065890 \\
\hline 7.00 & 0.779647485 & 0.779672021 & 0.000024536 & 0.076704282 & 0.076798600 & 0.000094318 \\
\hline 7.50 & 0.818035301 & 0.818047647 & 0.000012346 & 0.076743741 & 0.076788504 & 0.000044763 \\
\hline 8.00 & 0.856459307 & 0.856539607 & 0.000080300 & 0.077198620 & 0.077252941 & 0.000054321 \\
\hline 8.50 & 0.895323455 & 0.895367021 & 0.000043566 & 0.078101437 & 0.078111436 & 0.000009999 \\
\hline 9.00 & 0.934568610 & 0.934663389 & 0.000094779 & 0.078968134 & 0.078976002 & 0.000007868 \\
\hline 9.50 & 0.973697786 & 0.973698680 & 0.000000894 & 0.075002707 & 0.075018486 & 0.000015779 \\
\hline 10.00 & 1.000000000 & 1.000000000 & 0.000000000 & 0.000000000 & 0.000000000 & 0.000000000 \\
\hline
\end{tabular}




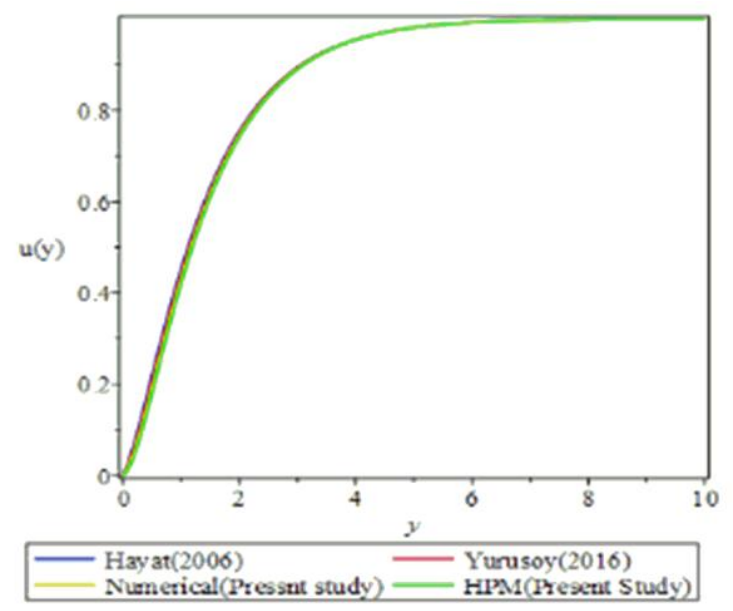

Fig. 2. Comparison of HPM solutions and Runge-Kutta solution with Hayat [19] an Yurusoy [18].

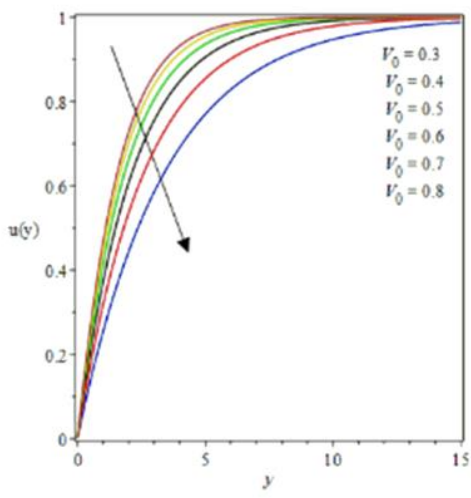

Fig. 3. Velocity profiles for varying $V_{0}$.

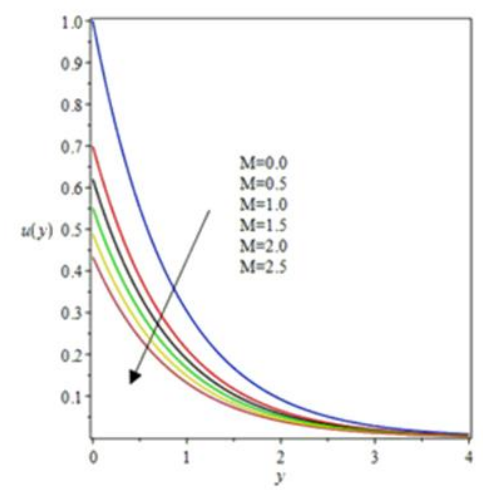

Fig. 5. Velocity profiles for varying M.

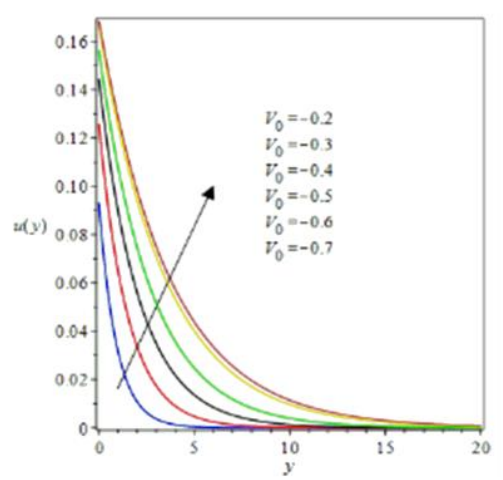

Fig. 4. Velocity profiles for varying $-V_{0}$.

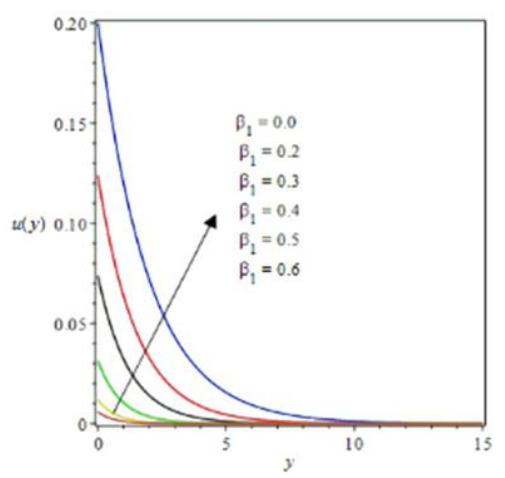

Fig. 6. Velocity profiles for varying $\beta_{1}$. 


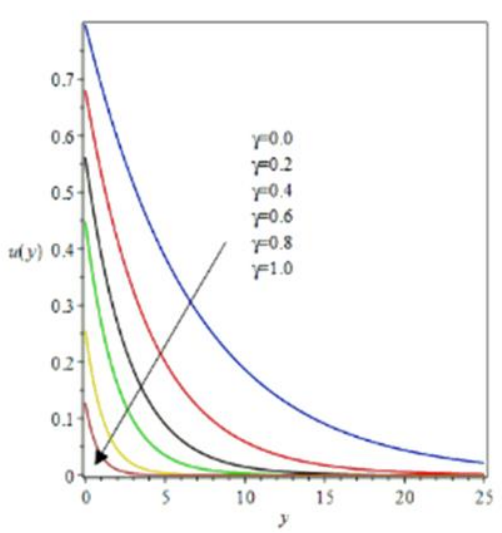

Fig. 7. Velocity profiles for varying $\gamma$.

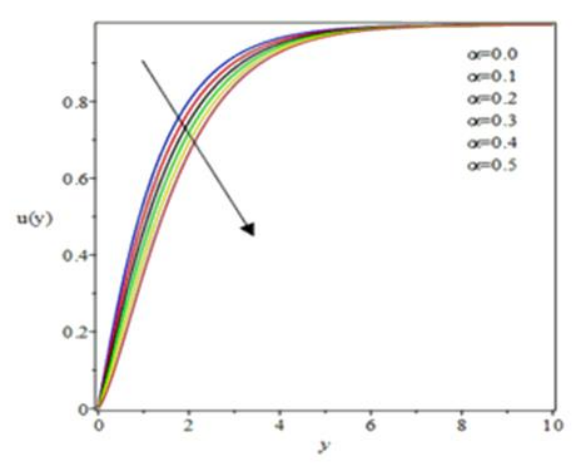

Fig. 8. Velocity profiles for varying $\alpha$.

\section{Conclusion}

This work investigates and analyses a mathematical model and solution for an unsteady Magnetohydrodynamic fourth grade fluid flow over a vertical plate in a porous medium with magnetic and suction/injection parameters effects. The impact of dimensionless second, third and fourth grade parameters with the effects of magnetic field and suction/injection parameters on the velocity field are displayed graphically and discussed. The system resulted in a higher order nonlinear differential equations governing the flow model with some boundary conditions which were solved via Homotopy Perturbation Method and the results show that:

1. Velocity profile decreases with increase in suction parameter at the surface of the plate thereby decreasing the momentum boundary layer.

2. Injection increases the flow of the velocity at the surface of the plate because it enhances velocity distribution in the boundary layer.

3. The damping effect of the suction velocity on the fluid flow is due to boundary force of high influence viscosity which resisted the heated fluid flow towards the plate.

4. The presence of magnetic field reduces velocity throughout the boundary layer because the Lorentz force acts as a retarding force and significantly reduced the momentum boundary layer thickness.

5. Fluid velocity decreases with increase in second grade parameter.

6. Increase in third grade parameter decreases the velocity field due to thickening behavior of the fluid.

7. The velocity profile increases as the fourth grade parameter increases due to shear thinning property of the fluid.

8. As the distance from the plate increases, the fluid velocity increases which satisfy the boundary condition. 


\section{References}

1. S. M. Arifuzaman, M. S. Kham, A. A. Al-Mamum, S. K. Reza-E-Rabbi, P. Biswas, and I. Karim, J. King Saud Uni. Sci. 31, 1388 (2019). https://doi.org/10.1016/j.jksus.2018.12.009

2. K. R. Rajagopal, Archives Mech. 32, 867 (1980). https://doi.org/10.1016/S0001-2092(07)61350-7

3. S. M. Arifuzzaman, M. F. U. Meheed, A. Al-Mamun, P. Biswas, and M. S. Khan, Int. J. Heat Technol. 36, 936 (2017). https://doi.org/10.18280/ijht.360321

4. A. M. Siddiqui, M. Ahmed, and Q. K. Ghori, Acta Mechanica 180, 117 (2005). https://doi.org/10.1007/s00707-005-0260-0

5. T. Hayat, T. H. A. Kara, and E. Momoniat, Math. Comp. Model. 41, 1347 (2005). https://doi.org/10.1016/j.mcm.2004.01.010

6. T. Hayat, H. Mambili-Mamboundou, and F. M. Mahomed, Nonlinear Analysis: Real World Applicat. 10, 368 (2009). https://doi.org/10.1016/j.nonrwa.2007.11.001

7. T. Hayat, H. Mambili-Mamboundou, C. M. Khalique, and F. M. Mahomed, Nonlinear Anal., Real World Applicat. 10, 3413 (2009). https://doi.org/10.1016/j.nonrwa.2008.09.030

8. S. K. Reza-E-Rabbi, S. M. Arifuzzaman, T. Sarkar, M. S. Khan, and S. F. Ahmmed J. King Saud Uni. Sci. 32, 690 (2020). https://doi.org/10.1016/j.jksus.2018.10.017

9. S. M. Arifuzzaman, M. S. Khan, M. F. U. Meheedi, B. M. J. Rana, and S. F. Ahmmed, Eng. Sci. Technol. Int. J. 21, 215 (2018). https://doi.org/10.1016/j.jestch.2018.03.004

10. R. L. Fosdick and K. R. Rajagopal, Arch. Ration. Mech. Anal. 70, 145 (1979). https://doi.org/10.1007/BF00250351

11. A. Passerini and M. C. Patria, Int. J. Nonlinear Mechanics 35, 1081 (2000). https://doi.org/10.1016/S0020-7462(99)00081-5

12. S. M. Arifuzzaman, M. S. Khan, M. S. Islam, M. M. Islam, B. M. J. Rana, P. Biswas, and S. F. Ahmmed. Front. Heat Mass Transfer, Thermal Fluid Central 9, 1 (2017).

13. J. H. He, Appl. Math. 135, 73 (2003). https://doi.org/10.1023/A:1023451224552

14. M. G. Sobamowo and A. T. Akinshilo, J. Mol. Liquids 241, 188 (2017). https://doi.org/10.1016/j.molliq.2017.05.145

15. S. N. Arifuzzaman, S. M. Khan, K. E. Hossain, M. S. Islam, S. Akter, and R. Roy, Front. Heat Mass Transfer, Thermal Fluid Central 9, 1 (2017). https://doi.org/10.5098/hmt.9.5

16. W. Tan and T. Masuoka, Int. J. Non-linear Mech. 40, 515 (2005). https://doi.org/10.1016/j.ijnonlinmec.2004.07.016

17. Y. Wang and W. Wu, Int. J. Non-Linear Mech. 42, 432 (2007). https://doi.org/10.1016/j.ijnonlinmec.2007.01.003

18. M. Yususoy, Math. Comput. Sci. 1, 29 (2016).

19. T. Hayat, S. Noreen, and M. Majid, Int. J. Therm. Sci. 47, 591 (2006). https://doi.org/10.1016/j.ijthermalsci.2007.05.005

20. J. H. He, Math. Eng. 178, 257 (1999). https://doi.org/10.1016/S0926-860X(99)00012-5

21. V. Marinca, N. Herisanu, C. Bota, and B. Msarinca, Appl. Math. Lett. 22, 245 (2009). https://doi.org/10.1016/j.aml.2008.03.019

22. S. Islam, Z. Bano, L. Siddique, and A. M. Siddqui, Comput. Math. Appl. 61, 1507 (2011). https://doi.org/10.1016/j.camwa.2011.01.014

23. S. Nadeem, T. Hayat, S. Abbasbandy, and M. Ali, Non-Linear Anal: Real World Appl. 11, 856 (2010). https://doi.org/10.1016/j.nonrwa.2009.01.030 